\title{
Invalidity of Meixner's Theorem in Irreversible Thermodynamics
}

\author{
R. E. Nettleton
}

(May 25, 1962)

\begin{abstract}
Meixner's theorem, on the preservation of the Onsager symmetry of phenomenological coefficients under transformations of forces and fluxes which leave the rate of entropy production invariant, is shown to be invalid under the restriction that all fluxes be even or odd under time-reversal and that the phenomenological equations be valid for very short times. This result extends an earlier proof of Coleman and Truesdell, whose proof does not impose these two restrictions. A new formulation of Meixner's theorem is thus shown to be required, and this is written down together with a statement of Onsager's theorem which is consistent with it.
\end{abstract}

\section{Introduction}

DeGroot has proposed ${ }^{1}$ that the theory of irreversible processes should rest on a fundamental postulate which, from the manner in which it has been used by deGroot and others, can be summarized by the following statement:

Statement I: Suppose $S$ is the entropy of a thermodynamic system and $\dot{S}$ the positive definite part $^{2}$ of its rate of change with respect to time $t$, and also that $X_{a}(a=1, \ldots, n)$, called "forces," are a set of linearly independent functions of the thermodynamic variables such that $\dot{S}$ can be written in the form $^{3}$

$$
\dot{S}=\sum_{a=1}^{n} J_{a} X_{a}
$$

where $J_{a}$ is called the "flux conjugate to $X_{a}$." Then the statement

$$
J_{a}=\sum_{b=1}^{n} L_{a b} X_{b}
$$

implies, in the absence of a magnetic field, that

$$
L_{a b}=L_{b a}
$$

if ${ }^{4} \quad X_{a}(t)=X_{a}(-t)$ and $X_{b}(t)=X_{b}(-t)$ or $X_{a}(t)=$ $-X_{a}(-t)$ and $X_{b}(t)=-X_{b}(-t)$. In the first of

\footnotetext{
is R. deGroot, Thermodynamics of Irreversible Processes, North-Holland Publishing Company, Amsterdam, 1951; see pp. 8, 216.

${ }_{2}^{2}$ In open systems, it is usually possible to express the total time rate of change of entropy as the sum of two terms, one of which reduces with the aid of the phenomenological laws of heat conduction, diffusion, and viscous dissipation, to a positive definite quadratic form. The other term is recognizable as an exchange of entropy with the surroundings which, in the theory of continuou systems [ref. 1, p. 98] appears as the divergence of an entropy flow vector.

${ }_{3}$ In the present statement no requirement is placed on the quantities $X$ other than that they be linearly independent and satisfy eq (1). It will be the purpose of the present paper to show that these requirements are inadequate for the truth of the statement and to establish the additional conditions which must be placed on the forces and on the phenomenological coefficients $L_{a b}$ if the statement is to be so formulated as to have general validity.
}

these two cases, $X_{a}$ and $X_{b}$ are said to be symmetric with respect to time inversion, and in the second case antisymmetric. Also

$$
L_{a b}=-L_{b a}
$$

if $X_{a}$ or $X_{b}$ is symmetric and the other of the two antisymmetric.

Coleman and Truesdell ${ }^{5}$ have shown that Statement I, the content of which is widely accepted as a valid statement of the Onsager-Casimir reciprocity theorem, goes beyond existing proofs of the theorem which assume that the forces $X_{a}$ are derivatives $\partial S / \partial y_{a}$ of $S$ with respect to a set of state variables, and that the flux $J_{a}$ conjugate to $X_{a}$ is equal to the time-derivative $\dot{y}_{a}$. These authors (see footnote 5) have pointed out that it is common to apply Statement I in cases where the $X_{a}$ are gradients of concentration or fluid velocity, which are types of forces for which it has never been proved in complete generality. ${ }^{6}$ In fact, they have shown that, without additional restrictions on the forces $J_{a}$ and fluxes $X_{a}$ beside eq (1) and the requirement of linear independence, Statement I is indeed false. To establish its invalidity, it suffices to assume the existence of $n$ pairs of functions $X_{a}, J_{a}$ for which eqs (1 to 4) hold, and then to find $n$ new pairs $J_{a}^{*}, X_{a}^{*}$ which are linear combinations of the $J_{a}$ and $X_{a}$ such that eqs (1) and (2) hold when all quantities therein are starred, while eqs (3) and (4) cease to hold for the matrix $\varepsilon^{*}=\left(L_{a b}^{*}\right)$ relating the $J_{a}^{*}$ to the $X_{b}^{*}$.

Such a transformation to new forces and fluxes violates a widely accepted statement, called "Meixner's theorem," which asserts that a transformation of this type cannot be found. This assertion goes beyond what is claimed by Meixner himself, who has defined ${ }^{7}$ a particular class of linear transforma-

${ }_{4} X_{a}\left((t)\right.$ is a notation which refers to an ensemble average $\left\langle X_{a}(\mathbf{P}, \mathbf{Q})\right\rangle$ of som ${ }^{4} X_{a}((t)$
function of the coordinates $\mathbf{Q}$ and momenta $\mathbf{P}$ of all the molecules in the system. function of the coordinates $\mathbf{Q}$ and momenta $\mathbf{P}$ of all the molecules in the system.
The transformation $t \rightarrow-t$ means reversal of the signs of the momenta inside the The transformation

angular bracket.

B. D. Coleman and C. Truesdell, J. Chem. Phys. 33, 28 (1960)

${ }^{6}$ Statement I has been shown to hold, by S. R. deGroot and P. Mazur, Phys. Rev. 94, 218, 224 (1954), in special cases of heat conduction and diffusion, but this demonstration cannot be automatically extended to cases where gradients of temperature or concentration are coupled in the phenomenological equations with forces of the type $\partial S / \partial y_{0}$

7.J. Meixner and H. G. Reik, Handbuch der Physik, Vol. 3, part 2, Ed. by S. Flügge, Springer Verlag, Berlin, 1959; see pp. 433, 434. 
tions from one set of fluxes $J_{a}$ and forces $X_{a}$ to another set $J_{a}^{*}$ and $X_{a}^{*}$ which have the property that if eqs ( 1 to 4 ) hold for the $J_{a}, X_{a}$, then these equations continue to hold when all quantities are starred. Transformations with this property are said to keep $\dot{S}$ and the Onsager symmetry invariant and are called symmetry-conserving transformations. From the existence of this class of transformations, it is commonly inferred, incorrectly, that all linear transformations which keep $\dot{S}$ invariant are also symmetry-conserving. The latter statement is the broad statement of "Meixner's theorem," which Meixner himself [compare ref. 7] realized could not be true in complete generality, although he did not discuss, as we shall do here, the various types of transformations which contradict it. Thus it has been left to Coleman and Truesdell (see footnote 5) to describe one set of transformations which keeps $\dot{S}$ invariant but is not symmetry-conserving, and which leads to counter-examples for Statement I.

These counter-examples (see footnote 5) are obtained by a transformation to new fluxes $J_{a}^{*}$, given by

$$
J_{a}^{*}=J_{a}+\sum_{b} W_{a b} X_{b}
$$

where $W_{a b}=-W_{b a}$ and it is assumed that any or all elements in the matrix $\left(W_{a b}\right)$ are $\neq 0$. The antisymmetry of $\left(W_{a b}\right)$ assures that if $X_{a}^{*} \equiv X_{a}$, then $\sum_{a} \cdot J_{a}^{*} X_{a}^{*}=\sum_{a} J_{a} X_{a}$. From this it readily follows (see footnote 5), without regard to whether the $X_{a}$ are all odd (antisymmetric) or all even (symmetric) or a mixture of the two, that the $J_{a}^{*}$ and $\dot{X}_{a}^{*}$ can satisfy conditions (1) and (2) of Statement I, under circumstances such that eq (3) does not obtain. For if $L_{a b}=L_{b a}$, then $L_{a b}^{*} \equiv L_{a b}+W_{a b} \neq L_{b a}^{*}$ when $W_{a b} \neq 0$, and we need say nothing explicitly about the timereversal symmetry of $X_{a}$ and $X_{b}$. Consequently, so long as any element of $\left(W_{a b}\right)$ can be taken $\neq 0$, the question of time-reversal symmetry of the fluxes and forces is irrelevant to the arguments of Coleman and Truesdell (see footnote 5).

However, we should note that if no prior restriction is placed on the elements of $\left(W_{a b}\right)$ and in particular we proceed to take them all $\neq 0$, then it is evident that if $J_{a}$ initially was definitely odd or even with respect to reversal of all the particle momenta, this will not be necessarily true of all the fluxes $J_{a}^{*}$. For if $J_{a}$ is odd and some of the $X_{b}$ are even, then $J_{a}^{*}$ will be a sum of odd and even terms, which itself has no definite time-reversal symmetry. Thus the transformation which leads to a counter-example for the broad statement of Meixner's theorem also produces fluxes which are neither symmetric or antisymmetric with respect to time inversion.

It is of interest, therefore, to examine the consequence of requiring that the fluxes and forces should all be even or odd, in order to determine whether this additional restriction is sufficient to assure that eqs (1) and (2) imply eqs (3) and (4). This restriction was indeed satisfied by the particular class of transformations considered by Meixner (see footnote 7), and it was taken by him to be a sufficient condition for the validity of "Meixner's theorem." Meixner's discussion [ref. 7, eq 8.3 and 8.4], however, assumes that the fluxes and forces are independent of one another, so that if $\dot{S}=$ $\sum_{a} J_{a} X_{a}=\sum_{a} J_{a}^{\prime} X_{a}$, then $J_{a}^{\prime}=J_{a}$. Since $J_{a}$ and $J_{a}^{\prime}$ are actually related to the $X_{a}$ by the phenomenological relations, Meixner's conclusion is not necessarily valid, and it is pertinent to reopen the question.

The particular physical motivation for this is that in a number of papers ${ }^{8}$ designed to extend irreversible thermodynamics to include inertial effects involving very short times and very high sound frequencies, Statement I has been applied to phenomenological relations in which all quantities had a definite time-reversal symmetry [thus satisfying Meixner's restriction (see footnote 7)]. 'These phenomenological equations also have the property, which we shall see is crucial for the application of Onsager's theorem, that concentration and velocity gradients are not linked directly to any of the fluxes conjugate to such gradients. The latter restriction corresponds to the inertial principle that a fluctuation from chemical or thermal equilibrium does not instantaneously cause the conjugate reaction rate or heat flux, respectively, to differ from zero; and this may be expressed by setting $L_{a b}=0$ when $X_{a}$ and $X_{b}$ are both even. For example, if $X_{a}$ and $X_{b}$ are proportional to concentration gradients in a multicomponent mixture, then the diffusion flux $J_{a}$ of component $a$ does not depend directly on $X_{a}$ or $X_{b}$, in accordance with the inertial principle that a concentration gradient does not produce an instantaneous flow.

In the present paper, we shall demonstrate that these additional restrictions on the phenomenological equations are indeed insufficient to assure the validity of Statement I. In fact, we must also require that all forces to which we seek to apply Onsager's theorem belong to one of two classes, of the type $\partial S / \partial y_{a}$ on the one hand, or concentration, velocity, or temperature gradients on the other, but no force can be a combination of the two types. This new restriction, together with limitations on the matrix $\left(L_{a b}\right)$ imposed by the principles of inertia and irreversibility, will be formulated in a Statement II given at the end of the paper.

The proof of the new statement is fairly trivial, and the main body of the paper is devoted to showing that such additional restrictions on the types of forces are necessary. This is done by finding transformations from a set $J_{a}, X_{a}$ which satisfy eqs (1 to 4) to a set $J_{a}^{\prime}, X_{a}^{\prime}$ which do not do so, even though all the forces are definitely even or odd, and $L_{a b}=0$ when $X_{a}$ and $X_{b}$ are both even. It will be seen that the arguments of Coleman and Truesdell are not applicable in this case, and that the transformations to new forces and fluxes, $J_{a}^{\prime}$ and $X_{a}^{\prime}$, must be constructed in a more elaborate manner to be discussed in the following two sections.

${ }^{8}$ R. E. Nettleton, J. Chem. Phys. 33, 237 (1960); Phys. Fluids 3, 216 (1960) Phys. Fluids 4, 74 (1961); Phys. Fluids 4, 1488 (1961). 


\section{Formulation of Problem}

In our construction of transformations which violate Meixner's theorem, we shall adopt conventional notation [ref. 1, p. 216] and denote even forces, $X_{i}(t)=X_{i}(-t)$, and their conjugate fluxes by Roman subscripts and odd forces, $X_{\alpha}(t)=-X_{\alpha}(-t)$, and their corresponding fluxes by Greek subscripts. The ?-matrix, in this notation, can be expressed in terms of four submatrices in the form

$$
\mathcal{R}=\left[\begin{array}{ll}
\left(L_{i j}\right) & \left(L_{i \alpha}\right) \\
\left(L_{\alpha i}\right) & \left(L_{\alpha \beta}\right)
\end{array}\right]
$$

where $L_{i \alpha}$ is the matrix element relating $J_{i}$ to $X_{\alpha}$ in the phenomenological eq (2), and the other submatrices are similarly defined. We shall assume that $\&$ has the symmetry suggested by Onsager's theorem, i.e.,

$$
L_{i j}=L_{j i}, \quad L_{\alpha \beta}=L_{\beta \alpha}, \quad L_{i \alpha}=-L_{\alpha i}
$$

and we shall consider what happens to this symmetry under transformations to new fluxes $J_{i}^{*}$ and forces $X_{i}^{*}$ which are such that

$$
\dot{S}=\sum J_{i} X_{i}+\sum_{\alpha} J_{\alpha} X_{\alpha}=\sum_{i} J_{i}^{*} X_{i}^{*}+\sum_{\alpha} J_{\alpha}^{*} X_{\alpha}^{*}
$$

i.e., which keep $S$ invariant under the transformation. Coleman and Truesdell (see footnote 5) have shown that a transformation of the type

$$
\begin{aligned}
& X_{i}^{*} \equiv X_{i}, \quad X_{\alpha}^{*} \equiv X_{\alpha} \\
& J_{i}^{*} \equiv J_{i}+\sum_{j} W_{i j} X_{j}+\sum_{\alpha} W_{i \alpha} X_{\alpha} \\
& J_{\alpha}^{*} \equiv J_{\alpha}+\sum_{j} W_{\alpha j} X_{j}+\sum_{\beta} W_{\alpha \beta} X_{\beta}
\end{aligned}
$$

satisfies eq (7) provided the matrix $(W)$ is antisymmetric, i.e., $W_{i j}=-W_{j i}, \quad W_{\alpha \beta}=-W_{\beta \alpha}$, and $W_{i \alpha}=-W_{\alpha i}$. It is also clear that (8) will destroy the symmetry given by eq (6), since the transformed $\imath$-matrix, $\imath^{*}$, has the property

$$
L_{i j}^{*}=L_{i j}+W_{i j} \neq L_{j i}^{*} .
$$

Let us remark here, however, that ordinarily $J_{i}$ has a definite time-reversal symmetry opposite to that of its conjugate force $X_{i}$. For example, this is the case when the flux is a rate of change, e.g., $\dot{y}_{a}$, of one of the variables on which the second depends linearly - the case usually discussed in proofs of Onsager's theorem [compare ref. 1, pp. $6,7]$. But although we may have $J_{i}(t)=-J_{i}(-t)$, we can still assign no definite time-reversal symmetry to $J_{i}^{*}$ given by eq (8) unless $W_{i j}=0$ for all pairs of Roman indices $i, j$. Similar considerations show that if $J_{\alpha}(t)=J_{\alpha}(-t)$, then $J_{\alpha}^{*}$ will be neither symmetric nor antisymmetric unless $W_{\alpha \beta}=0$ for all
Greek indices $\alpha, \beta$. But if $W_{i j}=0=W_{\alpha \beta}$ for all pairs $(i, j)$ and $(\alpha, \beta)$, then $L_{i j}^{*}=L_{i j}=L_{j i}^{*}, \quad L_{\alpha \beta}^{*}=L_{\alpha \beta}$ $=L_{\beta \alpha}^{*}$, and $L_{i \alpha}^{*}=L_{i \alpha}+W_{i \alpha}=-L_{\alpha i}^{*}$, so that the symmetry given by eq (6) is retained by the transformed R-matrix. Consequently, the transformation proposed by Coleman and Truesdell will not destroy the Onsager symmetry of the R-matrix if it does not destroy the time-reversal symmetry of the fluxes $J_{i}$.

It is for this reason that, as we remarked in the previous section, the Coleman-Truesdell demonstration is of no use in judging the applicability of Statement I to cases where the fluxes as well as the forces are all either odd or even with respect to time-reversal. The type of transformation which does prove useful for this purpose is one in which the fluxes $J_{i}^{*}$ are sums of fluxes $J_{i}$ and the $J_{\alpha}^{*}$ depend only on the $J_{\alpha}$, and similarly for the forces. If this can be done in such a way that eqs (1) and (2) hold for the transformed fluxes and forces when all quantities in these equations are starred, and yet eq (3) does not hold, then we shall have shown that Statement I must be modified before Onsager's theorem can be applied to the thermodynamic rate equations which have been derived (see footnote 8) to describe inertial effects in fluids. There the fluxes and forces all have a definite time-reversal symmetry, and temperature and concentration gradients are coupled to forces of the type $\partial S / \partial y_{a}$, so that existing proofs (see footnotes 1,6) of Onsager's theorem do not hold automatically.

Our desire to discuss inertial effects is also the reason we cannot invert the phenomenological equations and solve for the forces $X_{i}$ in terms of the fluxes $J_{i}$. If this could be done, the result could be substituted into eq (8) with $W_{i \alpha}=0$, and the Coleman-Truesdell transformation would reduce to an expression relating $J_{i}^{*}$ to a linear sum of fluxes $J_{i}$. There would then be nothing further to demonstrate by considering the transformation introduced in the following section [eq (10)].

However, if we are going to describe extremely short time intervals or high acoustic frequencies, we cannot have a phenomenological equation of the type which can be inverted, having the form

$$
J_{i}=\sum_{j} L_{i j} X_{j}
$$

in which $L_{i \alpha}=0=L_{\alpha i}$, and the $X_{\alpha}$ do not appear. For if the motion is suddenly reversed, the righthand member of this equation would be unchanged, while the flux $J_{i}$ would reverse its sign. This could be true only if $J_{i}=0$ at very high frequencies. Since it is the high-frequency components of the Fourier expansion of the motion which determine the behavior of the system over very short time intervals, the conclusion $J_{i}=0$ at high frequencies implies that the phenomenological equations give no short-time response to the imposition of thermodynamic forces, and thus they do not have an essential property of equations which include inertial effects. Consequently, we never have all the matrix elements $L_{\alpha i}=0$ when we describe the very short-time behavior of a physical system. This, in turn, means 
that it is impossible in general to solve for $X_{i}$ in terms of the fluxes $\left\{J_{k}\right\}$ alone or for $X_{\alpha}$ in terms of the fluxes $\left\{J_{\beta}\right\}$. Inversion of the phenomenological equations describing inertial effects expresses each force as a sum of fluxes some of which will always have the same time-reversal symmetry as the force in question. Substitution of the result into eqs (8) with $W_{i j} \neq 0$ leads to a $J_{i}^{*}$ which depends linearly on both $J_{i}$ and $J_{\alpha}$. Thus for at least one class of phenomenological equations which are of physical interest, i.e., those which include inertial effects, there is an essential difference between the transformations discussed in the present paper and those of Coleman and Truesdell.

\section{New Proof of Coleman-Truesdell Thecrem}

In the present section we shall discuss a very wide class of transformations which have the property that they conserve time-reversal symmetry of all fluxes and forces. It will be shown that transformations with this property may be so constructed that, while they preserve the antisymmetric Onsager relation, so that $L_{i \alpha}^{*}=-L_{\alpha i}^{*}$, nevertheless they lead to $L_{\alpha \beta}^{*} \neq L_{\beta \alpha}^{*}$. In accordance with the discussion of the preceding section, we shall assume $L_{i \alpha} \neq 0$ for some $i$ and $\alpha$, so that we have in mind the phenomenological description of inertial effects, and so that the transformations here proposed which conserve time-reversal symmetry cannot be reduced to the form assumed by Coleman and Truesdell. Furthermore, we shall take $L_{i j}=0$ for all Roman indices $i, j$, since, as was pointed out in the introduction, this is always a property of the phenomenological equations which describe inertial effects. This gives the appearance of restricting the proof to a very special class of phenomena. However, the description of inertial effects is the only existing instance in the scientific literature in which a solid physical basis has been established for the existence of antisymmetric reciprocal relations, and so the assumption $L_{i j}=0$ for all $i$ and $j$ appears physically to be quite general under circumstances such that some $L_{i \alpha} \neq 0$.

The particular transformations which we shall discuss are the following:

$$
\begin{array}{ll}
X_{i}^{*}=\sum_{j} A_{i j} X_{j}, & X_{\alpha}^{*}=\sum_{\beta} A_{\alpha \beta} X_{\beta} \\
J_{i}^{*}=\sum_{j} B_{i j} J_{j}, & J_{\alpha}^{*}=\sum_{\beta} B_{\alpha \beta} J_{\beta} .
\end{array}
$$

This assures that $X_{i}^{*}, J_{\alpha}^{*}$ are even functions of time and $X_{\alpha}^{*}, J_{i}^{*}$ odd functions, provided this was true of the corresponding unstarred quantities. We define the matrices $\mathfrak{A}, \mathfrak{B}$, and $\mathfrak{E}$, by the equations

$$
\begin{gathered}
\mathfrak{U} \equiv\left[\begin{array}{cc}
\left(A_{i j}\right) & 0 \\
0 & \left(A_{\alpha \beta}\right)
\end{array}\right] \quad \mathfrak{B} \equiv\left[\begin{array}{cc}
\left(B_{i j}\right) & 0 \\
0 & \left(B_{\alpha \beta}\right)
\end{array}\right] \\
\mathfrak{U} \equiv \mathfrak{B}^{\prime-1}(\mathfrak{S}+\mathfrak{C})
\end{gathered}
$$

where the prime denotes the transposed matrix, and $\xi$ is the identity. This definition assumes that the matrix $\mathfrak{B}$ is nonsingular, which is always possible since $\mathfrak{B}$ is arbitrary.

On substituting eqs (10) into eq (7) and using the phenomenological relations between fluxes and forces to express $\dot{S}$ as a quadratic form depending orly on the forces $X_{i}$ and $X_{\alpha}$, we readily find that eq (7) can hold if, and only if,

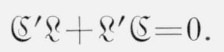

At the same time, eqs (10) imply that the transformed R-matrix is

$$
\mathfrak{R}^{*}=\mathfrak{B} \mathfrak{R} \mathfrak{U}^{-1}=\mathfrak{B} \mathfrak{R}(\mathfrak{\Im}+\mathfrak{C})^{-1} \mathfrak{B}^{\prime}
$$

provided $\mathfrak{C}$ is chosen so that $\mathfrak{A}$ is nonsingular. From eq (14) we readily see that the symmetry properties of $\mathfrak{R}^{*}$ are identical with those of $\mathfrak{R}(\mathfrak{\xi}+$ (5) $)^{-1}$, which we shall now examine.

$R$ is uniquely decomposable into the sum of a symmetric matrix $\&_{s}$ and an antisymmetric matrix $\varepsilon_{a}$, where

$$
\mathfrak{R}_{s}=\left[\begin{array}{cc}
\left(L_{i j}\right) & 0 \\
0 & \left(L_{\alpha \beta}\right)
\end{array}\right] \quad \mathfrak{R}_{a}=\left[\begin{array}{cc}
0 & \left(L_{i \alpha}\right) \\
\left(L_{\alpha i}\right) & 0
\end{array}\right] .
$$

One can readily show that multiplication of $\varepsilon_{s}$ by $c 5$ or ${ }^{\prime}{ }^{\prime}$ results in a matrix of the same type, with zeros in all the entries designated by zeros in eq (15). Similarly for $\varepsilon_{a}$. Substitution of eq (15) into eq (13) then gives

$$
\begin{aligned}
& \mathfrak{C}^{\prime} \mathfrak{Z}_{a}-\mathfrak{R}_{a} \mathfrak{C}=0 \\
& \mathfrak{C}^{\prime} \mathfrak{R}_{s}+\mathfrak{Z}_{s} \mathfrak{C}=0 .
\end{aligned}
$$

Equation (16) further implies

$$
\mathfrak{R}_{a}(\Im+\mathfrak{S})^{-1}=-\left(\Im+\mathfrak{C}^{\prime}\right)^{-1} \mathfrak{R}_{a}^{\prime} .
$$

From eq (14) we have:

$$
\mathfrak{B R}_{a}(\mathfrak{\Im}+\mathfrak{S})^{-1} \mathfrak{B}^{\prime}=\left[\begin{array}{cc}
0 & \left(L_{i \alpha}^{*}\right) \\
\left(L_{\alpha i}^{*}\right) & 0
\end{array}\right] .
$$

Equations (18) and (19) show that the transformation (10) preserves the antisymmetric Onsager relation $L_{i \alpha}^{*}=-L_{\alpha i}^{*}$. We can, however, find a solution of eqs (16) and (17) such that $L_{\alpha \beta}^{*} \neq L_{\beta \alpha}^{*}$, i.e., the Onsager symmetry is destroyed by the transformation. To this end, let us proceed to introduce the assumption that

$$
L_{i j}=0(\text { all } i, j) \text {. }
$$

This, as was previously pointed out, is the form of \&-matrix used in earlier papers on inertial effects (see footnote 8). When eq (20) is substituted into eq (17), we find the solution $\left(C_{i j}\right.$ and $C_{\alpha \beta}$ are elements of (5):

$$
C_{\alpha \beta}=\sum_{\gamma} W_{\alpha \gamma} L_{\gamma \beta}
$$

where $\left(W_{\alpha \beta}\right)$ is any antisymmetric matrix. From 
eq (16), we obtain

$$
\sum_{j} C_{j i} L_{j \beta}=\sum_{\gamma} L_{i \gamma} C_{\gamma \beta} .
$$

Equation (22) will, in general, possess a solution provided the number of indices $\beta$ does not exceed the number of Roman indices.

The existence of solutions of eqs (21) and (22), however, is, in general, imcompatible with the symmetry of the matrix

$$
\mathfrak{B R}_{s}(\mathfrak{\Im}+\mathfrak{C})^{-1} \mathfrak{B}^{\prime}=\left[\begin{array}{ll}
\left(L_{i j}^{*}\right) & 0 \\
0 & \left(L_{\alpha \beta}^{*}\right)
\end{array}\right] .
$$

If $\Omega_{s}(\Im+\mathcal{C})^{-1}$ equals its transpose, $\left(\Im+\mathcal{C}^{\prime}\right)^{-1} \Omega_{s}$, then eq (17) implies $\mathbb{R}_{s} \mathfrak{E}=0$, which is not satisfied by eq (21) for nonzero $\left(W_{\alpha \beta}\right)$.

A simple choice for $W_{\alpha \beta}$ may be made in the event, which frequently occurs in practice, that $\left(L_{i j}\right)$ and $\left(L_{\alpha \beta}\right)$ are both square matrices of the same even number $n \geq 2$ of rows [compare reference 8], so that $\left(C_{i j}\right)$ and $\left(C_{\alpha \beta}\right)$ are also n-rowed square matrices. In this case we can pick

$$
W_{\alpha \beta}=\epsilon_{1}\left(1-\delta_{\alpha \beta}\right) \operatorname{sgn}(\beta-\alpha)
$$

where $\delta_{\alpha \beta}$ is a Kronecker delta. Then from eqs (21) and (22),

$$
\operatorname{det} \mathcal{C}=\left[\operatorname{det}\left(C_{\alpha \beta}\right)\right]^{2}=\left\{\epsilon_{1}^{n} \operatorname{det}\left[\left(L_{\alpha \beta}\right)\right]\right\}^{2}
$$

provided the matrix $\left(L_{i \beta}\right)$ is nonsingular. When $\left(L_{i j}\right)=0$, the latter condition must hold in order that the fluxes $J_{i}(i=1, \ldots, n)$ be linearly independent. There must also exist cases in which, at the same time, det $\left(L_{\alpha \beta}\right) \neq 0$, since this determinant (see footnote 8 ) is the product of the relaxation frequencies for the inertial effects. Thus there will exist solutions of eqs (21) and (22) for which det (5 has an arbitrary positive value. The same is easily shown to hold for $\operatorname{det}(\Im+\mathfrak{S})$.

The latter result in turn assures that we can find a transformation such that the $J_{i}^{*}, J_{\alpha}^{*}$ are linearly independent, and similarly the $X_{i}^{*}, X_{\alpha}^{*}$. For the matrix $\mathfrak{B}$ is arbitrary, and thus can be chosen to be nonsingular, and then from eq (12)

$$
\operatorname{det} \mathfrak{U}=(\operatorname{det} \mathfrak{B})^{-1} \operatorname{det}(\mathfrak{\Im}+\mathfrak{C})
$$

which can be made $\neq 0$ by proper choice of $\epsilon_{1}$.

Thus we have established that starting with a linearly independent set of forces and fluxes, $J_{i}$, $J_{\alpha}$, and $X_{i}, X_{\alpha}$, with an \&-matrix which satisfies eq (16) and has the properties found to hold (see footnote 8) in a phenomenological theory that includes inertial effects, i.e., $L_{i j}=0$ and $L_{i \alpha} \neq 0$ for some $i$ and $\alpha$, we can transform to a new linearlyindependent set, $J_{i}^{*}, J_{\alpha}^{*}$ and $X_{i}^{*}, X_{\alpha}^{*}$, having definite time-reversal symmetry and such that $L_{\alpha \beta}^{*} \neq L_{\beta \alpha}^{*}$. This shows that Meixner's theorem does not hold in general even under these circumstances, although the Coleman-Truesdell proof does not apply here, and so we must seek a modification of Statement I.

\section{Reformulation of Meixner's Theorem}

It is evident from existing proofs of Onsager's theorem [ref. 1, chap. 2 and 11] that if there exists a set of symmetric state variables $y_{i}\left(i=1, \ldots, n_{1}\right)$, and antisymmetric variables $y_{\alpha}\left(\alpha=1, \ldots, n_{2}\right)$ such that $X_{i}=\partial S / \partial y_{i}, \quad J_{i}=\dot{y}_{i} \quad($ all $i)$, while $X_{\alpha}=\partial S / \partial y_{\alpha}$, $J_{\alpha}=\dot{y}_{\alpha}($ all $\alpha)$, and there are no nonzero forces except these, then Statement I is true. Furthermore, Statement I remains true if we transform from one set of fluxes and forces of this type to another set of the same type, and so Meixner's theorem is also true under this additional restriction.

The situations where Meixner's theorem has been found to fail arise where some or all of the forces $X_{\alpha}$ are not entropy derivatives of the form $\partial S / \partial y_{\alpha}$. For example, it follows from eq (12) that whenever the $\mathbb{R}$-matrix has the right properties so that $\mathfrak{E}$ does not vanish identically, then we can have a transformation [including an additional set of equations not shown in which the Greek subscripts $\alpha, \beta$ are replaced by Roman subscripts $i, j]$ :

$$
J_{\alpha}^{*} \equiv \dot{y}_{\alpha}^{*}=\sum_{\beta} B_{\alpha \beta} \dot{y}_{\beta}, \dot{y}_{\beta} \equiv J_{\beta}
$$

and

$$
X_{\alpha}^{*}=\partial S / \partial y_{\alpha}^{*}+\sum_{\beta, \gamma}(B)_{\beta \alpha}^{-1} C_{\beta \gamma} X_{\gamma}
$$

where

$$
\begin{aligned}
y_{\alpha}^{*}=\sum_{\beta} B_{\alpha \beta} y_{\beta}, \text { and } \partial S / \partial y_{\alpha}^{*} & =\sum_{\beta}\left(\partial S / \partial y_{\beta}\right)\left(\partial y_{\beta} / \partial y_{\alpha}^{*}\right) \\
& =\sum_{\beta}\left(B^{-1}\right)_{\beta \alpha} X_{\beta} .
\end{aligned}
$$

The transformed forces and fluxes of this type indeed satisfy eqs (7) and (10), and yet we have seen that $L_{\alpha \beta}^{*} \neq L_{\beta \alpha}^{*}$.

At this point we must not lose sight of the fact that in order to obtain a nonzero matrix $\left(C_{\alpha \beta}\right)$, and thus to find a counter-example to Meixner's theorem under the restriction to transformations of the form of eq (10), we had to assume $\left(L_{i j}\right)=0$, which has thus far been shown to hold in general only for the phenomenological equations which describe inertial effects (see footnote 8). In the latter case, when the system is a small subvolume immersed in an infinite fluid [compare ref. 8], we have to deal with two types of forces. First of all, there are the forces of the type $\partial S / \partial y_{i}$ and $\partial S / \partial y_{\alpha}$ which are functions of the state variables $y_{i}$ and $y_{\alpha}$ on which $S$ depends explicitly. Secondly, there are forces such as temperature, velocity, and concentration gradients on which $S$ does not depend explicitly and which are coupled in the phenomenological equations to forces which are of the type $\partial S / \partial y_{i}$ or $\partial S / \partial y_{\alpha}$. This is a state of affairs which arises because over very short times the system evolves independently of its environment, and it is only after one or more molecular collision times that the effect of gradients of temperature or velocity begins to be felt. Thus the entropy does not depend explicitly on these gradients, but instead on the variables $y_{i}$ and $y_{\alpha}$ which are related to the gradients through the phenomenological equations [compare ref. 8]. 
Because of the existence of these two types of forces, we can construct forces $X_{\alpha}^{*}$ which are not of the type $\partial S / \partial y_{\alpha}^{*}$ by adding gradients of velocity to the forces $\partial S / \partial y_{\alpha}$ as well as by combining the latter among themselves. Thus, in general, we cannot expect that $L_{\alpha \beta}=L_{\beta \alpha}$ if either $X_{\alpha}$ or $X_{\beta}$ involves any velocity, concentration, or temperature gradients which do not appear explicitly in $S$, although $L_{\alpha \beta}$ is equal to $L_{\beta \alpha}$ whenever $J_{\alpha}=\dot{y}_{\alpha}$, $J_{\beta}=\dot{y}_{\beta}, \quad X_{\alpha}=\partial S / \partial y_{\alpha}, \quad$ and $X_{\beta}=\partial S / \partial y_{\beta}$. In other words, it is possible, by combining forces of the first type (gradients) with forces of the second type $\left(\partial S / \partial y_{i}\right.$, etc.) to construct transformations which violate Meixner's theorem.

In addition to the demonstration that

(a) transformations can be found which keep $\dot{S}$ invariant, but which lead to an $\&$-matrix in which $L_{\alpha \beta} \neq L_{\beta \alpha}$, if, and only if, not all transformed fluxes are time-derivatives $\dot{y}_{i}, \dot{y}_{\alpha}$ or not all transformed fluxes are entropy derivatives $\partial S / \partial y_{i}, \partial S / \partial y_{\alpha}$, we have shown that

(b) if eqs (6) hold for a particular choice of $J_{i}$, $X_{i}, J_{\alpha}, X_{\alpha}$, then the antisymmetric relation $L_{i \alpha}=$ $-L_{\alpha i}$ is conserved by any transformation of the form of eq (10) which keers $\dot{S}$ invariant, without further restriction on the $J_{i}, X_{i}$ and $J_{\alpha}, X_{\alpha}$.

These two observations provide a new statement of Meixner's theorem.

Since the broad (and incorrect) statement of Meixner's theorem referred to in the introduction has often been advanced as a foundation for statement I of Onsager's theorem, we might jump to the conclusion that statements (a) and (b) of the reformulated Meixner's theorem are sufficient to provide the basis for a revision of Statement I. However, statement (b) does not provide for transformations more general in form than eq (10). Specifically, it omits transformations in which $J_{i}^{*}$ is a linear combination of forces $X_{\alpha}$ in addition to fluxes $J_{i}$. Such transformations preserve the timereversal symmetry of the transformed fluxes, although they are more general than eq (10). In order to cover all possible cases, it appears to be necessary to require:

(1) $\&$ must be the matrix of a positive definite quadratic form.

(2) Only those transformations are considered for which $L_{i j}^{*}=0$ (all $\left.i, j\right)$.

These physical restrictions are related respectively to the definition of irreversibility and to the inertial properties of mechanical systems [compare ref. 8]. When they obtain, they imply trivially that $L_{i \alpha}^{*}=$ $-L_{\alpha i}^{*}$, since otherwise $\dot{S}$ would contain cross terms of the type $X_{i}^{*} X_{\alpha}^{*}$ and no term in $\left(X_{i}^{* 2}\right)$, so that for $X_{i}^{*}$ sufficiently large, reversal of the signs of the forces $X_{\alpha}^{*}$ could make $\dot{S}<0$.

Actually, as we have already remarked at the beginning of the third section, condition (2) that $L_{i j}^{*}=0$ is probably superfluous, since this condition is always satisfied by phenomenological equations describing inertial effects, and, in the latter case, no transformation can be found which violates it.
Furthermore, no physical examples except the inertial rate equations have ever been demonstrated for which $L_{i \alpha} \neq 0$ for some $i$ and $\alpha$. Nevertheless, we shall provide for the possible existence of cases where $L_{i \alpha}$ $\neq 0 \neq L_{i j}$ in Statement II. There we shall require specifically that $\left(L_{i j}\right)=0$ whenever we extend Onsager's theorem to the coefficients which couple gradient-type forces to forces of the type $\partial S / \partial y_{a}$.

On the basis of these observations, we can restate Onsager's theorem in the following form, which covers the case in which there are fluxes which are not time-derivatives of state variables, or forces which are not derivatives of $S$ with respect to associated state variables:

Statement II: Suppose the entropy $S$ in a thermodynamic system is a function of a set of $N$ state variables, ${ }^{9}$ of which $n_{1}$ variables $y_{i}(i=1, \ldots$, $\left.n_{1}\right)$ are symmetric with respect to time-reversal and $n_{2}=N-n_{1}$ state variables $y_{\alpha}\left(\alpha=1, \ldots, n_{2}\right)$ are antisymmetric. With these variables we associate the forces $X_{i}, X_{\alpha}$ and fluxes $J_{i,} J_{\alpha}$ given by ${ }^{10}$

$$
\begin{gathered}
J_{i} \equiv \dot{y}_{i}, X_{i} \equiv \partial S / \partial y_{i}\left(i=1, \ldots, n_{1}\right) \\
J_{\alpha} \equiv \dot{y}_{\alpha}, X_{\alpha} \equiv \partial S / \partial y_{\alpha}\left(\alpha=1, \ldots, n_{2}\right) .
\end{gathered}
$$

Suppose also that $\dot{S}$, which denotes the irreversible part of the time-rate of change of $S$, is given by

$$
\dot{S}=\sum_{i=1}^{m_{1}} J_{i} X_{i}+\sum_{\alpha=1}^{m_{2}} J_{\alpha} X_{\alpha}
$$

where $m_{1} \geq n_{1}$ and $m_{2} \geq n_{2}$, and the $m_{1}$ quantities $J_{i}$ and $m_{2}$ quantities $J_{\alpha}$ are all linearly independent, and similarly for the $X_{i}, X_{\alpha}$. This provides for the case where there may be forces such as temperature, velocity, or concentration gradients on which $S$ does not depend explicitly, these being denoted by subscripts $n_{1}<i \leq m_{1}, n_{2}<\alpha \leq m_{2}$. It is assumed that the $X_{i}\left(1 \leq i \leq m_{1}\right)$ are all symmetric with respect to time-reversal and the $X_{\alpha}\left(1 \leq \alpha \leq m_{2}\right)$ are all antisymmetric. Then if

$$
\begin{aligned}
& J_{i}=\sum_{j=1}^{m_{1}} L_{i j} X_{j}+\sum_{\alpha=1}^{m_{2}} L_{i \alpha} X_{\alpha} \\
& J_{\alpha}=\sum_{j=1}^{m_{1}} L_{\alpha j} X_{j}+\sum_{\beta=1}^{m_{2}} L_{\alpha \beta} X_{\beta}
\end{aligned}
$$

we may assert, in the absence of a magnetic field, that

$$
\begin{aligned}
& L_{i j}=L_{j i}\left(i \leq n_{1}, j \leq n_{1}\right) \\
& L_{\alpha \beta}=L_{\beta \alpha}\left(\alpha \leq n_{2}, \beta \leq n_{2}\right) \\
& L_{i \alpha}=-L_{\alpha i}\left(i \leq n_{1}, \alpha \leq n_{2}\right.
\end{aligned}
$$

and for all $i, \alpha$ whenever $L_{i j}=0$ for all $i, j$ ).

\footnotetext{
${ }^{9}$ It must be remembered here that these $N$ variables are not necessarily the entire set of state variables appearing in the phenomenological equations, since we have pointed out that there may be others on which $S$ does not depend ex plicitly. These additional variables, which are usually gradients, characterize the state of the surroundings of the system, and they affect the rates of change of the variables $y_{i}, y_{\alpha}$, even though the entropy and free energy depend only on the latter.

In an open system $\dot{y}_{i}$ and $\dot{y}_{\alpha}$ denote only those parts of the time-rates of change which are due to irreversible processess ular diffusion across the boundary.
} 


\section{Discussion}

The last result in Statement II expresses the fact, which has already been mentioned, that when $L_{i j}=0$ (all $i, j$ ), we must have $L_{i \alpha}=-L_{\alpha i}$ in order to assure that $\dot{S}$ contains no cross terms $X_{i} X_{\alpha}$; for otherwise $\dot{S}$ would not be a positive definite quadratic form in the forces. The other two symmetric reciprocal relations, $L_{i j}=L_{j i}$ and $L_{\alpha \beta}=L_{\beta \alpha}$, involve only the forces associated with the $N$ state variables, and not the remaining $m_{1}+m_{2}-N$ forces, since there exists no proof of symmetric Onsager relations for the latter. Indeed we can find transformations involving the gradient-type forces which are such that Onsager symmetry as expressed by Statement I is violated. It has also been shown that transformations contradicting Statement I can be found even when the gradients all vanish, and so we have been careful in formulating Statement II to restrict those forces $X_{\alpha}$ and $X_{\beta}$ for which we assert $L_{\alpha \beta}=L_{\beta \alpha}$ to the ones which can be written in the form $\partial S / \partial y_{\alpha}$ and $\partial S / \partial y_{\beta}$. However, in the existing applications (see footnote 8) of Onsager's theorem to rate equations describing inertial effects, those forces which did not depend on gradients not appearing in $S$ were always so constructed as to be of the form $\partial S / \partial y_{i}, \partial S / \partial y_{\alpha}$. Therefore, it is only with respect to the presence of velocity, concentration (in binary mixtures), and temperature gradients that serious questions have arisen over past usage of Statement I.

If a concentration or temperature gradient should appear in the phenomenological equations multiplied by a coefficient of the type $L_{i j}$, then statement II would tell us nothing about the symmetry of this coefficient with respect to interchange of its indices. In previous applications (see footnote 8) it has always been possible to write phenomenological equations in such a form that this situation did not arise, and so that $L_{i j}=0$ for all $i, j$, thus providing no circumstances not covered by statement II. It appears that, if we take account of inertial effects in such processes as heat conduction and diffusion, then we can always avoid the presence of terms concerning which statement II says nothing, although no completely general demonstration of this apparent fact exists.

(Paper 66B3-79) 